\title{
Síntese e avaliação antifúngica do benzil 2,3-didesoxi-a-D-eritro-hex-2- enopiranosídeo contra espécies de Candida spp. e estudo da associação com anfotericina B
}

\author{
Synthesis and antifungal evaluation of benzyl 2,3-dideoxy-a-D-erythro-hex-2-enopyranoside against species of
} Candida spp. and study of the association with amphotericin $B$

Síntesis y evaluación antifúngica de bencil 2,3-didesoxi-a-D-eritro-hex-2-enopiranosido contra Candida spp. y estudio de la asociación con anfotericina $B$

Rodrigo Ribeiro Alves CAIANA ${ }^{1}$

Joyse Maria Braga dos SANTOS ${ }^{1}$

Josivan Pereira da Silva JÚNIOR ${ }^{1}$

Jadson de Farias SILVA ${ }^{2}$

Wylly Araújo de OLIVEIRA ${ }^{1}$

Juliano Carlo Rufino FREITAS ${ }^{1,2}$

${ }^{1}$ Centro de Educação e Saúde, Universidade Federal de Campina Grande - UFCG, Olho D'Agua da Bica, 58175-000, Cuité - PB, Brasil ${ }^{2}$ Departamento de Química, Universidade Federal Rural de Pernambuco - UFRPE, Campus Dois Irmãos - Bloco 2, 52171-900, Recife - PE, Brasil

\section{Resumo}

Introdução: O número crescente de infecções causadas por fungos e o surgimento de microrganismos resistentes tem sinalizado a necessidade da busca por novos agentes antifúngicos, fomentando a pesquisa em moléculas promissoras, como os $O$-glicosídeos. Objetivo: Partindo dessa premissa, objetivamos a síntese e investigação da atividade antifúngica do benzil 2,3-didesoxi- $\alpha$-D-eritro-hex-2-enopiranosídeo isolado e quando associado com a anfotericina B. Material e métodos: Inicialmente foi sintetizado o benzil 4,6-di- $O$-acetil-2,3-didesoxi- $\alpha$-D-eritrohex-2-enopiranosídeo a partir da reação entre o 3,4,6-tri-O-acetil-D-glucal e álcool benzílico via catálise ácida e irradiação ultrassônica seguida por sua hidrólise em meio básico para levar ao benzil 2,3-didesoxi- $\alpha$-D-eritro-hex-2-enopiranosídeo. Este foi submetido à avaliação antifúngica por meio do método da microdiluição em caldo e estudo da associação com a anfotericina B pelo método de Checkerboard Resultados: O benzil 4,6-di- $O$-acetil-2,3-didesoxi- $\alpha$-D-eritro-hex-2-enopiranosídeo e o benzil 2,3-didesoxi- $\alpha$-D-eritro-hex-2-enopiranosídeo foram obtidos em excelentes rendimentos 91 e $94 \%$, respectivamente. O benzil 2,3-didesoxi- $\alpha$-D-eritro-hex-2-enopiranosídeo apresentou atividade antifúngica apenas contra as cepas de Candida albicans ATCC 76615, Candida albicans ATCC 76485 e Candida guilliermondii LM 103, e sua associação com a anfotericina B foi classificada como indiferente. Conclusão: Estes resultados possibilitam futuros estudos envolvendo esta classe de moléculas, avaliação dos possíveis mecanismos de ação e avaliar outras atividades biológicas, uma vez que esta classe molecular sustenta a expectativa de baixa toxicidade, devido ao seu padrão de biocompatibilidade.

Descritores: Síntese Química; Metabolismo dos Carboidratos; Fungos; Candida.

\section{Abstract}

Introduction: The growing number of fungal infections and the emergence of resistant microorganisms have signaled the need to search for new antifungal agents, encouraging research in promising molecules such as $O$-glycosides. Objective: Based on this premise, we aimed to synthesize and investigate the antifungal activity of benzyl 2,3-dideoxy- $\alpha$-D-erythro-hex-2-enopyranoside alone and when associated with amphotericin B. Material and methods: Initially the benzyl 4,6-di- $O$-acetyl-2,3-dideoxy- $\alpha$-D-erythro-hex-2-enopyranoside was synthesized from the reaction between 3,4,6-tri- $O$-acetyl-D-glucal and benzyl alcohol via acid catalysis and ultrasonic irradiation followed by hydrolysis in basic media to give benzyl 2,3-dideoxy- $\alpha$-D-erythro-hex-2-enopyranoside. This was submitted to antifungal evaluation by means of the broth microdilution method and study of the association with amphotericin B by the Checkerboard method. Results: Benzyl 4,6-di- $O$-acetyl2,3-dideoxy- $\alpha$-D-erythro-hex-2-enopyranoside and benzyl 2,3-dideoxy- $\alpha$-D-erythro-hex-2-enopyranoside were obtained in excellent yields of 91 and 94\%, respectively. Benzyl 2,3-dideoxy- $\alpha$-D-erythro-hex-2-enopyranoside showed antifungal activity only against Candida albicans ATCC 76615, Candida albicans ATCC 76485 and Candida guilliermondii LM 103 strains, and its association with amphotericin B was classified as indifferent. Conclusion: These results enable future studies involving this class of molecules, evaluation of possible mechanisms of action and evaluate other biological activities, since this class molecule supports the expectation of low toxicity due to its biocompatibility pattern.

Descriptors: Chemical Synthesis; Carbohydrate Metabolism; Fungi; Candida.

\section{Resumen}

Introducción: El creciente número de infecciones fúngicas y la aparición de microorganismos resistentes ha señalado la necesidad de buscar nuevos agentes antifúngicos, alentando la investigación en moléculas prometedoras, como los $O$-glucósidos. Objetivo: En base a esta premisa, nuestro objetivo fue sintetizar e investigar la actividad antifúngica de las bencil 4,6-di-O-acetil-2,3-didesoxi- $\alpha$-D-eritro-hex-2enopiranosido aislado y cuando se asocia con la anfotericina B. Material y métodos: Bencil 4,6-di-O-acetil-2,3-didesoxi- $\alpha$-D-eritro-hex-2enopiranosido se sintetizó a partir de la reacción entre 3,4,6-tri- $O$-acetil-D-glucal y alcohol bencílico por catálisis e irradiación ultrasónica seguido de su hidrólisis en medio básico para conducir al bencil 2,3-didesoxi- $\alpha$-D-eritro-hex-2-enopiranosido. Se sometió a evaluación antifúngica por el método de microdilución en caldo y a la asociación con anfotericina B por el método Checkerboard. El bencil 4,6-di-Oacetil-2,3-didesoxi- $\alpha$-D-eritro-hex-2-enopiranosido y el benzil 2,3-didesoxi- $\alpha$-D-eritro-hex-2-enopiranosido obtenidos con excelentes rendimentos, 91 y 94\% respectivamente. Resultados: el bencil 2,3-didesoxi- $\alpha$-D-eritro-hex-2-enopiranosido mostró actividad antifúngica solo contra las cepas Candida albicans ATCC 76615, Candida albicans ATCC 76485 y Candida guilliermondii LM 103, y su asociación con la anfotericina B se clasificó como indiferente. Conclusión: Estos resultados permiten futuros estudios que involucren a esta clase de moléculas, la evaluación de posibles mecanismos de acción y la evaluación de otras actividades biológicas, ya que esta clase de moléculas respalda la expectativa de baja toxicidad debido a su patrón de biocompatibilidad.

Descriptores: Síntesis Química; Metabolismo De Los Hidratos De Carbono; Hongos; Candida.

\section{INTRODUÇÃO}

As infecções fúngicas invasivas têm sido um fator preocupante na saúde da população mundial devido suas consideráveis taxas de morbimortalidade, além dos elevados custos em saúde. O número de pessoas acometidas por infecções fúngicas vem crescendo nas últimas décadas, principalmente em pacientes imunocomprometidos ${ }^{1}$, recém-nascidos, idosos, diabéticos, pacientes no pós-operatório, mulheres grávidas ou que fazem uso de anticoncepcionais, além daqueles que estão em uso prolongado de antibióticos ou de corticoides ${ }^{2}$. Além disso, há uma diversidade de microrganismos 
resistentes à terapia antifúngica, reduzindo a quantidade de fármacos totalmente eficazes $\mathrm{e}$ interferindo no processo de tratamento da população em geral.

Entre 25 a $75 \%$ dos indivíduos saudáveis possuem Candida spp. na sua microbiota normal ${ }^{3}$. Entretanto, diferentes espécies deste gênero podem ser citadas como causadores de infecções fúngicas invasivas, sendo a $C$. albicans a espécie mais prevalente tanto em pacientes sadios como em indivíduos mórbidos ${ }^{1}$.

A C. albicans está presente em aproximadamente $70 \%$ dos isolados clínicos, contudo, sua incidência relativa nas infecções está em declínio devido ao aparecimento de outras espécies infeciosas, como $C$. glabrata, $C$. tropicalis e $C$. parapsilosis, as quais estão entre as mais frequentes causas de candidemia depois da $C$. albicans. ${ }^{1}$ Segundo Ashong et al. ${ }^{4}$, o índice de mortalidade relativo à candidemia oscila entre 31 e $44 \%$, um valor que tende a ampliar-se devido ao desenvolvimento de espécies mais resistentes, como $C$. glabrata e $C$. krusei.

As infecções por estes microrganismos são comumente encontradas na cavidade oral, vulvovaginal e urinária, as quais podem comprometer as mucosas e se alastrar para grandes órgãos do corpo através da corrente sanguínea levando a quadros infecciosos sistêmicos ${ }^{1,2}$.

Ademais, o desenvolvimento de resistência de muitos microrganismos a diversos antimicrobianos, inclusive os fármacos utilizados tradicionalmente como antifúngicos, tem preocupado os órgãos de saúde mundial ${ }^{2,5-8}$.

$\mathrm{O}$ uso indiscriminado dos antimicrobianos tem sido uma razão bem explícita para o desenvolvimento de uma diversidade de microrganismos resistentes ${ }^{9}$. A preocupação com os crescentes casos de resistência microbiana alastra-se mundialmente, uma vez que na ausência de descobertas significativas de antimicrobianos, estimase que surgirão doenças incuráveis pela tecnologia atual, podendo matar 10 milhões de pessoas até o ano de $2050^{10}$. Este problema emergente fomenta pesquisas que visam o desenvolvimento de novos fármacos antifúngicos que possam superar os fármacos atuais tanto em termos de eficácia relacionada à resistência ${ }^{9}$. Neste âmbito, a obtenção de moléculas via síntese orgânica vem se destacando em meio às distintas estratégias para obtenção de novos fármacos, uma vez que estas transformações químicas proporcionam a descoberta de moléculas com características adequadas de eficácia e segurança, assegurando, deste modo, um melhor perfil farmacodinâmico e farmacocinético ${ }^{11}$.

Desta forma, uma classe molecular interessante sinteticamente é a classe dos $O$-glicosídeos. Essas moléculas foram obtidas pela primeira vez por Emil Fischer em 1893 por meio da reação denominada glicosilação. Desde então diversos grupos de pesquisa concentram esforços a fim de melhorar o processo de obtenção destas moléculas (Figure 1) ${ }^{12-15}$.

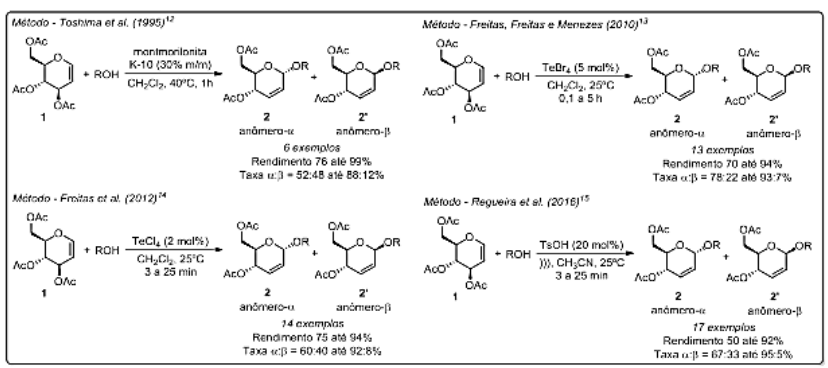

Figura 1: Exemplos de métodos para síntese dos $O$-glicosídeos ${ }^{12-15}$.

Os $O$-glicosídeos estão presentes em uma gama de produtos naturais, os quais apresentam importantes atividades farmacológicas, a saber: ação anti-inflamatória, antibiótica, antiviral, antitumoral, imunológica, anticoagulante e antiprotozoária ${ }^{16}$.

As características químicas favoráveis destes $O$-glicosídeos permitem que eles sejam facilmente submetidos a diferentes modificações estruturais ${ }^{17}$, favorecendo o seu emprego como intermediários sintéticos, como protótipos para o desenvolvimento de novas moléculas e como componentes estruturais de fármacos ${ }^{18}$.

Muitos destes glicosídeos são obtidos a partir da D-glicose, uma molécula altamente comum aos seres vivos e amplamente aplicada nos mais variados setores industriais, como o farmacêutico, alimentício, cosmético, químico, dentre outros ${ }^{19}$. Devido a este padrão de compatibilidade biológica da D-glicose, os glicosídeos obtidos apresentam a expectativa de baixa toxicidade, uma vez que uma alternativa para resolver os problemas de citotoxicidade consiste na utilização de substratos que apresentam compatibilidade com os sistemas biológicos ${ }^{20,21}$.

Junto a isso, nota-se que novas terapias, incluindo combinações de fármacos com diferentes mecanismos de ação, podem melhorar a eficácia dos tratamentos, justificando o fato de que os fármacos mais vendidos estão em maior parte em associação a outros princípios ativos. ${ }^{22}$ A flucitosina, por exemplo, é um antifúngico bastante utilizado em associação a outros agentes antifúngicos, que contribui para o progresso do tratamento e diminuição dos efeitos colaterais $^{23}$.

Assim, o presente trabalho teve como objetivo sintetizar e avaliar a atividade antifúngica do benzil 2,3-didesoxi- $\alpha$-D-eritro-hex-2-enopiranosídeo isolado e quando associado com a anfotericina $\mathrm{B}$ frente a diferentes espécies de Candida spp.

\section{MATERIAL E MÉTODO}

- Equipamentos e Análise dos Dados

As análises de ressonância magnética nuclear (RMN) foram realizadas em um espectrômetro 
VARIAN $^{\circledR}$ modelo Unity Plus-300 utilizando como solvente o clorofórmio deuterado $\left(\mathrm{CDCl}_{3}\right)$. Este espectrômetro foi calibrado usando tetrametilsilano $(0,00 \mathrm{ppm})$ como referência interna para os núcleos de ${ }^{1} \mathrm{H} \mathrm{e}^{13} \mathrm{C}$, e todas as constantes de acoplamento $(J)$ foram descritas em hertz $(\mathrm{Hz})$. O espectro de infravermelho (IV) foi registrado em um espectrofotômetro de infravermelho com transformada de Fourier no Spectrum 400 FT-IR/FTNIR Spectrometer modelo PerkinElmer, sendo a amostra preparada como pastilhas de $\mathrm{KBr}$, com número de scans de 16 , resolução 4 e número de onda de 4000 a $400 \mathrm{~cm}^{-1}$. A rotação específica foi determinada em um polarímetro digital da marca $\mathrm{JASCO}^{\circledR}$ P-2000 equipado com a luz de sódio em comprimento de onda $589 \mathrm{~nm}$. A amostra foi preparada em concentração de $1 \mathrm{~g} / \mathrm{mL}(\mathrm{em} \mathrm{MeOH})$ em uma cubeta de $1 \mathrm{~mL}$. A chapa de agitação com aquecimento e a manta aquecedora foram da marca Fisaton modelo 754A e 102E, respectivamente. O solvente foi removido utilizando um evaporador rotativo da Büchi modelo R-114 conectado a uma bomba de vácuo modelo KNF-Neuberger, e o solvente remanescente foi removido utilizando uma bomba de alto vácuo da Edwards modelo RV3.

\section{- Materiais, solventes e reagentes}

$\mathrm{O}$ monitoramento das reações foi realizado através da cromatografia em camada delgada (CCD) utilizando placas de sílica-gel contendo indicador fluorescente $\mathrm{F}_{254}$ da Merck. Para visualização, as placas foram colocadas em solução ácida (EtOH/ $\mathrm{H}_{2} \mathrm{SO}_{4}$ na proporção $95 / 5$, respectivamente). A purificação através da cromatografia líquida em coluna foi realizada utilizando sílica-gel 60 da Merck (70-230 mesh) como fase estacionária e sistemas de hexano:acetato de etila como fase móvel numa coluna de vidro em diferentes proporções. Os solventes comerciais foram purificados de acordo com os protocolos descritos na literatura. ${ }^{24} \mathrm{O}$ hexano e o acetato de etila foram destilados com coluna de vigreau $^{25}$, o diclorometano foi destilado sob hidreto de cálcio, e o metanol sob magnésio metálico e iodo sublimado. Os reagentes 3,4,6-tri- $O$-acetil-D-glucal e o álcool benzílico foram adquiridos da empresa Sigma Aldrich.

- Síntese do benzil 4,6-di-O-acetil-2,3-didesoxi- $\alpha$ D-eritro-hex-2-enopiranosídeo (2)

$\mathrm{O}$ benzil 4,6-di- $O$-acetil-2,3-didesoxi- $\alpha$-Deritro-hex-2-enopiranosídeo (2) foi sintetizado a partir da reação de glicosilação através do protocolo de Regueira et al. ${ }^{15}$ com algumas modificações. Em um balão de fundo redondo com capacidade para 50 $\mathrm{mL}$ foi preparada uma solução de 3,4,6-tri- $O$-acetil-D-glucal (1) $(0,544 \mathrm{~g} ; 2 \mathrm{mmol})$ e do álcool benzílico $(0,768 \mathrm{~g} ; 2,4 \mathrm{mmol})$ em acetonitrila $(8 \mathrm{~mL})$, depois foi adicionado o ácido $p$ toluenossulfônico (17 mg; $20 \%$ molar). A mistura foi colocada em um banho de ultrassom por 5 minutos.
Após esse período de tempo, a mistura reacional foi diluída com acetato de etila $(20 \mathrm{~mL})$ e lavada com uma solução saturada de $\mathrm{NaHCO}_{3}(3$ x $20 \mathrm{~mL})$ e solução saturada de $\mathrm{NaCl}(50 \mathrm{~mL})$. As fases orgânicas foram combinadas e secas com $\mathrm{MgSO}_{4} \mathrm{e}$ os solventes foram removidos sob pressão reduzida.

O produto bruto foi purificado através de cromatografia líquida em coluna, utilizando sílica-gel 60 como fase estacionária e sistemas hexano:acetato de etila como fase móvel numa coluna de vidro. A purificação em coluna do composto 2 iniciou usando apenas hexano, como solvente eluente, e terminou com a mistura de $95 \%$ de hexano e $5 \%$ de acetato de etila.

- Sintese do benzil 2,3-didesoxi- $\alpha$-D-eritro-hex-2enopiranosídeo (3)

$\mathrm{O}$ benzil 2,3-didesoxi- $\alpha$-D-eritro-hex-2enopiranosídeo (3) foi sintetizado a partir da hidrólise do composto 2 em meio básico, através do protocolo de Santos et $\mathrm{al}^{26}$ com algumas modificações. Desta forma, em um tubo de ensaio, o composto $2(0,320 \mathrm{~g} ; 1 \mathrm{mmol})$ foi dissolvido em metanol (4,0 mL). A esta solução foi adicionado o sal básico $\mathrm{K}_{2} \mathrm{CO}_{3}(0,276 \mathrm{~g} ; 2 \mathrm{mmol})$ e água $(1,2 \mathrm{~mL})$. A mistura reacional foi mantida sob agitação durante 5 minutos sob temperatura ambiente $\left(28 \pm 2^{\circ} \mathrm{C}\right)$. Após o término da reação, a mistura reacional foi transferida para um balão e o solvente foi removido sob pressão reduzida. Na sequência foi adicionado acetato de etila e $\mathrm{MgSO}_{4}$ anidro. Em seguida, foi realizada uma filtração simples e o filtrado coletado em um segundo balão, o qual foi evaporado sob pressão reduzida para retirada do solvente. O composto foi purificado através de cromatografia líquida em coluna, utilizando sílica-gel como fase estacionária e sistemas de hexano:acetato de etila como fase móvel numa coluna de vidro. A purificação em coluna do composto 3 iniciou usando apenas hexano, como solvente eluente, e terminou com a mistura de $60 \%$ de hexano e $40 \%$ de acetato de etila.

- Preparo da solução e cepas fúngicas

A solução foi preparada de acordo com as normas estabelecidas pelo método de referência para testes de diluição em caldo para determinação da sensibilidade de leveduras à terapia antifúngica, ${ }^{27}$ com algumas modificações. A solução do benzil 2,3didesoxi- $\alpha$-D-eritro-hex-2-enopiranosídeo (3) foi preparada com o auxílio de dimetilsulfóxido (DMSO). Foram utilizados controles com DMSO nas mesmas concentrações utilizadas nos testes.

Foram utilizadas dez cepas diferentes de Candida, sendo elas: C. albicans ATCC 40042, $C$. albicans ATCC 76615, C. albicans ATCC 76485, C. parapsilosis ATCC 22019, C. tropicalis ATCC 13803, C. krusei ATCC 6258, C. krusei 120, C. krusei LM 13, C. guilliermondii LM 301 e $C$. guilliermondii LM 103. 
No experimento de sensibilidade aos antifúngicos foi empregado um inóculo compreendendo, aproximadamente, $1-5 \times 10^{6}$ $\mathrm{UFC} / \mathrm{mL}$ com turvação correspondente ao tubo $0,5 \mathrm{da}$ escala de McFarland.

\section{- Determinação da Concentração Inibitória Mínima (CIM)}

A CIM foi determinada a partir da técnica de microdiluição em caldo, estabelecida conforme o método de referência para testes de diluição em caldo para determinação da sensibilidade de leveduras à terapia antifúngica. ${ }^{27}$ Foram utilizadas placas plásticas estéreis transparentes contendo 96 poços com o meio de cultura líquido RPMI-1640. As concentrações testadas do benzil 2,3-didesoxi- $\alpha$-Deritro-hex-2-enopiranosídeo oleosa

foram de $1024 \mu \mathrm{g} / \mathrm{mL}$ a $1 \mu \mathrm{g} / \mathrm{mL}$ em diluições seriadas 1:2, sendo $\mathrm{o}$ experimento conduzido com um inóculo de concentração final entre 1-5 x $10^{5} \mathrm{UFC} / \mathrm{mL}$ em cada cavidade. Todos os testes foram realizados em triplicata. Posteriormente, as placas foram incubadas em estufa a $35^{\circ} \mathrm{C}$ por 24 48 horas, sendo considerada como CIM, a menor concentração da substância capaz de inibir o crescimento visual das cepas utilizadas nos testes de sensibilidade. ${ }^{28}$

- Associação do benzil 2,3-didesoxi- $\alpha$-D-eritro-hex2-enopiranosídeo (3)

Para a realização da associação foram preparadas, em tubos separados, diferentes soluções do benzil 2,3-didesoxi- $\alpha$-D-eritro-hex-2enopiranosídeo (3) e da anfotericina nas concentrações CIMx8, CIMx4, CIMx2, CIM, CIM/2, $\mathrm{CIM} / 4$ e CIM/8. A associação foi realizada pelo método de Checkerboard para a determinação do Índice de Concentração Inibitória Fracionada (ICIF) em placas plásticas estéreis contendo 96 poços. As soluções das devidas sustâncias foram dispostas nas placas de modo que as concentrações do benzil 2,3didesoxi- $\alpha$-D-eritro-hex-2-enopiranosídeo dispostas na vertical, associassem com todas as concentrações da anfotericina $\mathrm{B}$, as quais foram dispostas de forma horizontal. Os ensaios foram realizados em triplicata. Foi utilizado um inóculo contendo $1-5 \times 10^{5} \mathrm{UFC} / \mathrm{mL}$ em cada uma das cavidades utilizando-se para o estudo as cepas de $C$. albicans ATCC 76615 e C. albicans ATCC 76485. Logo após a realização das associações, os ensaios foram encubados em estufa a aproximadamente 35 ${ }^{\circ} \mathrm{C}$, por 48 horas. A leitura dos resultados foi feita visualmente. O Índice de Concentração Inibitória Fracionada (ICIF) foi calculado de acordo com a literatura ${ }^{29}$.

Considerando que "A" seja o benzil 2,3didesoxi- $\alpha$-D-eritro-hex-2-enopiranosídeo (3) e "B" a anfotericina $\mathrm{B}$, cada valor de Concentração Inibitória Fracionada (CIF) foi calculado dividindo-se valor da CIM encontrado de cada substância isolada com a
CIM derivada da associação. A partir disso, a associação foi classificada como sinérgica (ICIF $\leq$ $0,5$ ), com aditismo (ICIF $>0,5 \mathrm{e}<1,0)$, indiferente $(1$ a 4) e antagônica $(\geq 4)^{30}$.

\section{RESULTADOS E DISCUSSÃO}

Nossa estratégia sintética é descrita na Figura 2. Inicialmente, o 3,4,6-tri- $O$-acetil-D-glucal (1) reagiu com o álcool benzílico empregando como catalisador o ácido $p$-toluenossulfônico $(\mathrm{TsOH})$ sob sonicação levando a mistura anomérica do benzil 4,6 di- $O$-acetil-2,3-didesoxi- $\alpha$-D-eritro-hex-2enopiranosídeo (2) e benzil 4,6-di- $O$-acetil-2,3didesoxi- $\beta$-D-eritro-hex-2-enopiranosídeo (2') em excelente rendimento $(91 \%)$ e boa seletividade anomérica (86:14). A mistura anomérica 2 e 2' foi purificada por coluna cromatográfica levando ao anômero $\alpha$ puro (2).

Posteriormente, foi realizado a hidrólise dos grupos acetilas do composto 2 utilizando o sal básico carbonato de potássio $\left(\mathrm{K}_{2} \mathrm{CO}_{3}\right)$ em meio hidrometanólico levando à formação do benzil 2,3didesoxi- $\alpha$-D-eritro-hex-2-enopiranosídeo (3) em excelente rendimento (94\%).

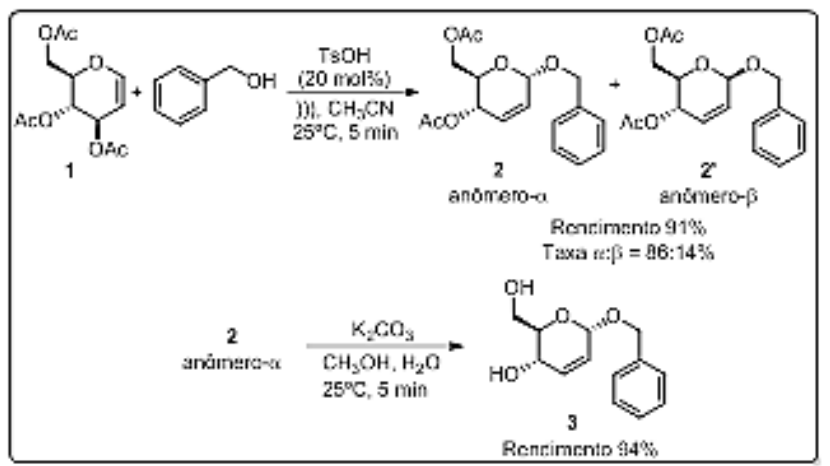

Figura 2: Estratégia sintética para síntese do composto 3.

O composto 2 foi obtido na forma oleosa, translúcida e nosso rendimento foi superior ao obtido por Melo et al..$^{31}$. Cabe destacar que neste trabalho, ${ }^{31}$ o grupo do professor Srivastava descreveu a síntese do composto 2 por dois métodos distintos, em que o primeiro consistiu no uso de agitação e aquecimento, enquanto que o segundo no uso da irradiação de micro-ondas. De acordo com os resultados do trabalho do professor Srivastava, o composto 2 foi obtido com $85 \%$ de rendimento após 180 minutos no primeiro método, enquanto que no segundo método o composto 2 foi obtido com $80 \%$ de rendimento após 4 minutos de reação ${ }^{31}$.

O composto 3 foi obtido na forma de um sólido branco e amorfo, com um rendimento de $94 \%$ em um tempo de 5 minutos. Este resultado é muito semelhante ao descrito no trabalho de Santos et al. ${ }^{26}$, os quais obtiveram esse composto 3 com rendimento de $95 \%$ após o mesmo tempo reacional.

Os compostos 2 e 3 obtidos foram caracterizados por diferentes técnicas. Os dados dessa caracterização estão descritos na Tabela 1. 
Tabela 1: Dados de caracterização dos compostos 2 e 3 .

Compostos

5

Dados de caracterização
$[\alpha]_{D}{ }^{20}+74,7$ (c $\left.1,00, \mathrm{MeOH}\right)$

$\mathrm{RMN}^{1} \mathrm{H}\left(300 \mathrm{MHz}, \mathrm{CDCl}_{3}\right): \delta 7,34-7,24(\mathrm{~m}, 5 \mathrm{H}$,

$\left.\mathrm{H}_{\text {aromático }}\right), 5,88(\mathrm{dl}, 1 \mathrm{H}, \mathrm{H}-3, J=10,5 \mathrm{~Hz}), 5,86$ -

$5,82(\mathrm{~m}, 1 \mathrm{H}, \mathrm{H}-2), 5,35-5,31(\mathrm{~m}, 1 \mathrm{H}, \mathrm{H}-4), 5,10$

(sl, $1 \mathrm{H}, \mathrm{H}-1), 4,78(\mathrm{~d}, 1 \mathrm{H}, \mathrm{OCH} 2(\mathrm{C} 6 \mathrm{H} 5), J=$

$12,0 \mathrm{~Hz}), 4,56$ (d, 1 H, OCH2(C6H5), $J=12,0$

$\mathrm{Hz}$ ), 4,25 (dd, $1 \mathrm{H}, \mathrm{H}-6, \mathrm{~J}=11,8$ e $5,4 \mathrm{~Hz}), 4,16$

(dd, $1 \mathrm{H}, \mathrm{H}-6$ ', $J=11,8$ e $2,4 \mathrm{~Hz}$ ), 4,13 (ddd,

$1 \mathrm{H}, \mathrm{H}-5, J=12,3,5,4$ e $2,4 \mathrm{~Hz}$ ), 2,09 (s, 3H, OAc), 2,07 (s, 3H, -OAc)

$\mathrm{RMN}^{13} \mathrm{C}\left(75 \mathrm{MHz}, \mathrm{CDCl}_{3}\right) \delta 170,8 ; 170,3$;

137,$6 ; 129,3 ; 128,5 ; 128,0 ; 127,9 ; 127,8 ; 93,6$ 70,$3 ; 67,1 ; 65,3 ; 62,9 ; 20,9 ; 20,8$ $[a]_{D}^{25}+31,7$ (c 1,00, MeOH)

IV $(\mathrm{KBr}) v_{\max } 3323,3031,2988,1454,1093$, $690 \mathrm{~cm}^{-1}$

$\mathrm{RMN}^{1} \mathrm{H}\left(300 \mathrm{~Hz}, \mathrm{CDCl}_{3}\right) \delta 7,37-7,26(\mathrm{~m}, 5 \mathrm{H}$

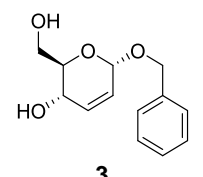

$\left.\mathrm{H}_{\text {aromático }}\right), 5,95$ (dl, $\left.1 \mathrm{H}, \mathrm{J}=9,9 \mathrm{~Hz}, \mathrm{H}-3\right), 5,76$

(dt, $1 \mathrm{H}, J=9,9$ e 2,4 Hz, H-2), 5,09 (sl, $1 \mathrm{H}, \mathrm{H}$

1), $4,19(\mathrm{dl}, 1 \mathrm{H}, \mathrm{H}-4), 4,77(\mathrm{~d}, 1 \mathrm{H}, J=12,3 \mathrm{~Hz}$ $\mathrm{OCH} 2(\mathrm{C} 5 \mathrm{H} 6)), 4,60(\mathrm{~d}, 1 \mathrm{H}, J=12,3 \mathrm{~Hz}$,

$\mathrm{OCH} 2(\mathrm{C} 5 \mathrm{H} 6)), 3,80(\mathrm{~d}, 2 \mathrm{H}, J=4,2 \mathrm{~Hz}, \mathrm{H}-6)$,

$3,73(\mathrm{dd}, 1 \mathrm{H}, \mathrm{J}=4,2 \mathrm{~Hz}, \mathrm{H}-5), 2,30(\mathrm{sl}, 2 \mathrm{H}$, $\mathrm{OH})$

$\mathrm{RMN}{ }^{13} \mathrm{C}\left(75 \mathrm{MHz}, \mathrm{CDCl}_{3}\right) \delta 137,7 ; 133,6$;

128,$5 ; 128,1 ; 127,9 ; 126,1 ; 93,7 ; 71,6 ; 70,4$; 64,$1 ; 62,5$.

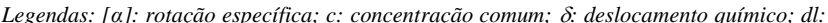
dupleto largo; J: constante de acoplamento; dt: dupleto de tripleto; dd: dupleto de dupleto; ddd: dupleto de dupleto de dupleto; m: miltipleto; sl: singleto largo; -OAc: grupo acetoxi; s: singleto; d: dupleto; $v_{\max }$ : número de ondas.

O composto 3 foi submetido aos ensaios de atividade antifúngica (Tabela 2). Neste estudo o composto 3 mostrou-se ativo contra as cepas de $C$. albicans ATCC 76615, C. albicans ATCC 76485 e C. guilliermondii LM 103, no entanto, não foi observada atividade contra as demais cepas nas concentrações utilizadas e o controle utilizando anfotericina B inibiu o crescimento de todas as cepas.

Tabela 2: Valores da CIM em $\mu \mathrm{g} / \mathrm{mL}$ das substâncias testadas

\begin{tabular}{c|c|c}
\hline \multirow{2}{*}{ Cepas } & \multicolumn{2}{|c}{ CIM $(\boldsymbol{\mu g} / \mathbf{m L})$} \\
\cline { 2 - 3 } & Composto 3 & Amfotericina B \\
\hline C. albicans ATCC 40042 & $>1024$ & 0,5 \\
\hline C. albicans ATCC 76615 & 1024 & 0,5 \\
\hline C. albicans ATCC 76485 & 1024 & 0,5 \\
\hline C. parapsilosis ATCC 22019 & $>1024$ & 0,25 \\
\hline C. tropicalis ATCC 13803 & $>1024$ & 0,25 \\
\hline C. krusei ATCC 6258 & $>1024$ & 0,25 \\
\hline C. krusei 120 & $>1024$ & 0,25 \\
\hline C. krusei LM 13 & $>1024$ & 0,25 \\
\hline C. guilliermondii LM 301 & $>1024$ & 0,25 \\
\hline C. guilliermondii LM 103 & 512 & 0,25 \\
\hline
\end{tabular}

Vale ressaltar que a terapia combinada pode prevenir o surgimento de microrganismos resistentes e também aumentar o espectro de ação dos agentes antimicrobianos devido a um efeito sinérgico. ${ }^{33}$ Além disso, estão surgindo cada vez mais cepas de Candida resistentes à anfotericina $\mathrm{B} .{ }^{34}$ Logo, a associação de diferentes princípios ativos pode aumentar a potência dos fármacos, reduzir a toxicidade e os efeitos adversos e superar a barreira da resistência. ${ }^{35}$ Desta forma, a tabela 3 demonstra o resultado da associação do composto $3 \mathrm{com}$ a anfotericina B para as cepas de C. albicans ATCC76615 e C. albicans ATCC 76485, uma vez que esta espécie está presente em mais 50\% dos isolados clínicos ${ }^{1}$.
Tabela 3: Associação do glicosídeo 2,3-insaturado e anfotericina B contra C. albicans.

\begin{tabular}{c|c|c|c|c}
\hline Cepas & $\begin{array}{c}\text { CIF } \\
\text { Composto 3 }\end{array}$ & $\begin{array}{c}\text { CIF } \\
\text { Anfotericina B }\end{array}$ & ICIF & Interpretação \\
\hline $\begin{array}{c}\text { C. } \\
\text { albicans } \\
\text { ATCC }\end{array}$ & 0,125 & 2,0 & 2,125 & indiferente \\
76615 & & 2,0 & 2,5 & indiferente \\
\hline $\begin{array}{c}\text { C. } \\
\text { albicans } \\
\text { ATCC } \\
76685\end{array}$ & 0,5 & & & \\
\hline
\end{tabular}

De acordo com a Tabela 3, os resultados da associação do composto $3 \mathrm{com}$ a anfotericina B, medicamento padrão-ouro para tratamento de infecções fúngicas invasivas ${ }^{32}$, foi classificada como indiferente, implicando que o composto 3 não interferiu na atividade da anfotericina B.

Vale destacar que o composto 3 é interessante biologicamente, pois pertence a classe dos $O$ glicosídeos, a qual vem ganhando destaque como promissores agentes terapêuticos no tratamento de diferentes distúrbios ${ }^{7}$. Por exemplo, estudos indicaram que glicosídeos poliacetilenos exibiram ação anti-inflamatória, antimicrobiana, anti-HIV e antialérgico ${ }^{34}$. Adicionalmente, Guo et al. ${ }^{36}$ isolaram alguns desses glicosídeos poliacetilenos com atividade antimicrobiana contra Bacillus anthracis.

Recentemente, nosso grupo de pesquisa descreveu uma interessante ação antitumoral de diferentes $O$-glicosídeos contendo uma unidade $Z$ enínica em sua estrutura, os quais diferem dos compostos 2 e 3 apenas em relação a sua unidade aglicona, conservando a porção sacarídica da molécula ${ }^{37}$.

Também foi avaliada a atividade antimicrobiana de glicosídeos de isoflavonas contra bactérias e fungos, demonstrando sua atuação semelhante à estreptomicina contra Escherichia coli e semelhante à griseofulvina contra o Aspergillus niger $^{38}$.

Em outro trabalho do nosso grupo de pesquisa, foi avaliada a atividade antifúngica do prop-2-in-1-il 4,6-di- $O$-acetil-2,3didesoxi- $\alpha$-D-eritro-hex-2-enopiranosídeo frente diferentes espécies de Candida não albicans, em que foi constatado um sinergismo desta molécula com a anfotericina B, sugerindo seu potencial para terapias combinadas $^{39}$. Vale destacar que essa molécula apresenta uma arquitetura molecular muito semelhante ao composto 3 , o que motiva futuras mudanças na estrutura do composto 3 a fim de acentuar a sua atividade.

Dessa forma, torna-se evidente a importância da pesquisa de novas alternativas para a terapia combinada a fim melhorar as propriedades farmacocinéticas e farmacodinâmicas de antimicrobianos já existentes e, principalmente, erradicar a evolução do surgimento de microrganismos resistentes. Assim como, oferecer uma melhor terapia para a população em geral, com a 
inovação de fármacos combinados que possam diminuir os efeitos colaterais provocados por estes agentes.

$\mathrm{O}$ resultado aqui apresentado configura-se como o primeiro passo no desenvolvimento de novos fármacos, fornecendo informações importantes que auxiliarão na condução de pesquisas futuras, uma vez que tais evidências apontam o composto 3 como um candidato promissor para o processo de otimização, com boa probabilidade de vir a ser útil futuramente na obtenção de bons fármacos antifúngicos.

CONCLUSÃO

O benzil 4,6-di- $O$-acetil-2,3-didesoxi- $\alpha$-Deritro-hex-2-enopiranosídeo (2) e o benzil 2,3-didesoxi- $\alpha$-D-eritro-hex-2-enopiranosídeo

foram sintetizados em excelente rendimento (91\% e 94\%, respectivamente) e suas estruturas foram confirmadas por diferentes técnicas espectroscópicas.

O composto 3 apresentou atividade antifúngica contra as cepas de C. albicans ATCC 76615, C. albicans ATCC 76485 e C. guilliermondii LM 103, porém, os valores de CIM foram elevados. Sua associação com a anfotericina B foi classificada como indiferente, ou seja, o composto 3 não interferiu na atividade da anfotericina B.

Adicionalmente, estes resultados possibilitam futuros estudos envolvendo modificações estruturais nesta classe de moléculas, avaliação do mecanismo de ação frente aos diferentes microrganismos patogênicos e avaliação de outras atividades biológicas, uma vez que esta classe molecular sustenta a expectativa de baixa toxicidade devido seu padrão de biocompatibilidade se configurando como um candidato promissor para o processo de otimização, com boa probabilidade de vir a ser útil futuramente na obtenção de bons fármacos antifúngicos.

\section{AGRADECIMENTOS}

\begin{tabular}{lrcr}
\hline \multicolumn{1}{c}{ Os } & autores & agradecem às agências & de \\
fomento & CNPq & $(434012 / 2018-1)$ & ao \\
PRONEM/FACEPE & (APQ-0476-1.06/14) & pelo
\end{tabular}
suporte financeiro, A CAPES e FACEPE (IBPG0094-1.06/17) pelas bolsas concedidas e a Central Analítica do DQF-UFPE pelas análises dos compostos.

\section{REFERÊNCIAS}

1. Silva $S$, Negri $M$, Henriques $M$, Oliveira $R$, Williams DW, Azeredo J. Candida glabrata, Candida parapsilosis and Candida tropicalis: biology, epidemiology, pathogenicity and antifungal resistance. FEMS Microbiol Rev. 2012;36:288-305.

2. Vieira AJH, Santos JI. Mecanismos de resistência de Candida albicans aos antifúngicos anfotericina
B fluconazol e caspofungina. RBAC. 2017;49:235-9.

3. De Rossi T, Lozovoy MAB, Silva RV, Fernandes EV, Geraldino TH, Costa IC, et al. Interações entre Candida albicans e Hospedeiro. Semina: Cienc Biol Saude. 2011;32:15-28.

4. Ashong CN, Hunter AS, Mansouri MD, Cadle RM, Hamill RJ, Musher DM. Adherence to clinical practice guidelines for the treatment of candidemia at a Veterans Affairs Medical Center. Int J Health Sci. 2017;11:18-23.

5. Kung HC, Huang PY, Chen WT, Ko BS, Chen YC, Chang SC, et al. 2016 guidelines for the use of antifungal agents in patients with invasive fungal diseases in Taiwan. $\mathbf{J}$ microbiol Immunol infect. 2018;51:1-17.

6. Patil S, Rao RS, Raj AT, Sanketh DS, Sarode S, Sarode G. Oral Candidal Carriage in Subjects with Pure Vegetarian and Mixed Dietary Habits. J Clin Diagn Res. 2017;11:22-4.

7. Khan H, Khan Z, Amin S, Mabkhot YN, Mubarak MS, Hadda TB, et al. Plant bioactive molecules bearing glycosides as lead compounds for the treatment of fungal infection: A review. Biomed Pharmacother. 2017;93:498-509.

8. Guzzetti LB, Cecília M, Vescina M, Gil F, Gatti BM. Candidemias en pediatría: distribución de especies y sensibilidad a los antifúngicos. Rev Argent Microbiol. 2017;49:320-2.

9. Wiederhold NP. Antifungal resistance: current trends and future strategies to combat. Infect Drug Resist. 2017;10:249-59.

10. World Health Organization. WHO/EMP/IAU/2017.11. 2017. Acesso em: 04 Fev 2019. Disponível em: <www.who.int/medicines/areas/rational_use/antib acterial_agents_clinical_development/en/>.

11. Lúcio Neto MP. Avaliação tóxica, citotóxica, genotóxica e mutagênica do composto 3-(2-cloro6-flurobenzil) - imidazolidina-2,4-diona em células eucarióticas. Teresina. Dissertação [Mestrado em Ciências Farmacêuticas] Universidade Federal do Piauí; 2011.

12. Toshima K, Ishizuka T, Matsuo G, Nakata M. Practical Glycosidation Method of Glycals Using Montmorillonite $\mathrm{K}-10$ as an Environmentally Acceptable and Inexpensive Industrial Catalyst. Chem Ver. 1995;4:306-8.

13. Freitas JCR, Freitas Filho JR, Menezes PH. Stereoselective Synthesis of 2,3-unsaturated-OGlycosides promoted by TeBr4. J Braz Chem Soc. 2010;21:2169-72.

14. Freitas JCR, Couto TR, Paulino AAS, Freitas Filho JR, Malvestiti I, Oliveira RA, et al. Stereoselective synthesis of pseudoglycosides catalyzed by $\mathrm{TeCl} 4$ under mild conditions. Tetrahedron. 2012;68:8645-54. 
15. Regueira JLLF, Dantas CR, Freitas JJR, Silva AJFS, Freitas Filho JR, Menezes PH, et al. Stereoselective Synthesis of 2,3-Unsaturated Pseudoglycosides Promoted by Ultrasound. Synthesis. 2016;48:1069-78.

16. Moura AL, Lima LMA, Bezerra GB, Freitas JJR, Belian MF, Ramos CS, et al. O-glicosídeos 2,3insaturados: aplicações, rearranjo de ferrier e reações. Quím Nova. 2018;41:550-66.

17. Dantas CR. Síntese estereosseletiva e caracterização estrutural de compostos Z-eniínicos acoplados a pseudoglicosídeos. Recife. Dissertação [mestrado em química] Universidade Federal Rural de Pernambuco; 2017.

18. Nigudkar SS, Demchenko A,V. Stereocontrolled 1,2-cis glycosylation as the driving force of progress in synthetic carbohydrate chemistry. Chem Sci. 2015;6:2687-704.

19. Silva RO, Freitas Filho JR, Freitas JCR. DGlicose, uma Biomolécula Fascinante: História, Propriedades, Produção e Aplicação. Rev Virt Quim. 2018;10:875-91.

20. Delbianco M, Bharate P, Varela-Aramburu S, Seeberger PH. Carbohydrates in Supramolecular Chemistry. Chem Rev. 2016;116:1693-752.

21. Lima JACL, Mata MMS, Silva RG, da Silva WE, Freitas JCR, Freitas Filho JR. Avanços Recentes na Química de C-Glicosídeos: Aplicaçao, Síntese e Reaçoes. Rev. Virt. Quim. 2018;10:900-39.

22. Silva AF, Silva DA. Fármacos anti-inflamatórios não esteroidais mais dispensados em uma farmácia comercial do município de itaocara, estado do Rio de Janeiro, Brasil. Acta Biomed Bras. 2012;3:1-14.

23. Costa AOC. Estudo in vitro e in silico da atividade antifúngica dos isômeros r-(+) e s-()itronelal sobre fungos do gênero Cryptococcus. João Pessoa. Dissertação [Mestrado em Produtos Naturais e Sintéticos Bioativos] - Universidade Federal da Paraíba; 2017.

24. Perrin DD, Amarego WLF. Purifications of laboratory chemicals. 3 ed., Pergamon Press: Oxford, 1996.

25. Santos CS, Dos Santos RS, Silva RO, Freitas Filho JR, Freitas JCR. Uma acetilação eficiente e econômica do 1,2:3,4-di-O-isopropilideno- $\alpha$-Dgalactopiranose utilizando anidrido acético ativado in situ pela argila montmorilonita K10. Ceramica. 2018;64:616-22.

26. Santos JAM, Santos CS, Almeida CLA, Silva TDS, Freitas Filho JR, Militão GCG, et al. Structure-based design, synthesis and antitumoral evaluation of enulosides. Eur $\mathrm{J}$ Med Chem. 2017;128:192-201.

27. Clinical and Laboratory Standards Institute. M27A3: reference method for broth dilution antifungal susceptibility testing of yeasts; approved standard. 3rd ed. Wayne: Clinical and Laboratory Standards Institute; 2008.

28. Oliveira WA, Pereira FO, Luna GCDG, Lima IO, Wanderley PA, Lima RB, et al. Antifungal activity of Cymbopogon winterianus jowitt ex bor against Candida albicans.. Braz J Microbiol. 2011;42:433-41.

29. Pippi B. Avaliação da capacidade de Candida parapsilosis e Candida glabrata desenvolverem resistência fenotípica à própolis vermelha brasileira e ao fluconazol e avaliação de sua atividade antifúngica em associação com fluconazol e anidulafungina. Porto Alegre. Dissertação [Mestrado em Microbiologia Agrícola e do Ambiente] - Instituto de Ciências Básicas da Saúde, Universidade do Rio Grande do Sul; 2014.

30. Doern CD. When Does 2 Plus 2 Equal 5? A Review of Antimicrobial Synergy Testing. J clin microbial. 2014;52:4124-8.

31. Melo ACN, Oliveira RN, Freitas Filho JR, Silva T G, Srivastava RM. Synthesis of anti-inflammatory 2,3-unsaturated O-glycosides using conventional and microwave heating techniques. Heterocycl Commun. 2017;23:205-11.

32. Lima B. Anfotericina B pré-aquecida: avaliação da atividade frente a isolados clínicos de Candida spp. do HU-UFSC. Florianópolis. Dissertação [Mestrado em Farmácia] - Universidade de Santa Catarina; 2017.

33. Cavalcanti IMF, Menezes TGC, Campos LAA, Ferraz MS, Maciel MAV, Caetano MNP, et al. Interaction study between vancomycin and liposomes containing natural compounds against methicillin-resistant Staphylococcus aureus clinical isolates. Braz J Pharm Sci. 2018;54:20310.

34. Targino CSPC. Estudo da atividade antifúngica e dos mecanismos de ação do peptídeo Ctn[15-34], um fragmento C-terminal da crotalicidina, derivado de uma catelicidina expressa nas glândulas de veneno de cascavéis. Fortaleza. Tese [Doutorado em Ciências Farmacêuticas] Faculdade de Farmácia, Odontologia e Enfermagem, Universidade Federal do Ceará; 2017.

35. Spader TB. Avaliação da suscetibilidade de Rhodotorula mucilaginosa frente a associações de antifúngicos com fármacos diversos. Porto Alegre. Tese [Doutorado em Ciências Pneumológicas] - Faculdade de Medicina, Universidade Federal do Rio Grande do Sul; 2017.

36. Guo J, Wang A, Yang K, Ding H, Hu Y, Yang Y, et al. Isolation, characterization and antimicrobial activities of polyacetylene glycosides from Coreopsis tinctoria Nutt. Phytochemistry. 2017;136:65-9. 
37. Dantas CR, de Freitas JJR, Barbosa QPS, Militão GCG, Silva TDS, da Silva TG, et al. Stereoselective Synthesis and Antitumoral Activity of Z-Enyne Pseudoglycosides Org Biomol Chem. 2016;14:6786-95.

38. Qing-Hu W, Na-Ren-Chao-Ke-Tu H, Na-Yin-Tai D, Xiu-Lan W, Wu-Li-Ji AA. The structural elucidation and antimicrobial activities of two isoflavane glycosides from Astragalus membranaceus (Fisch) Bge. var. mongholicus (Bge) Hsiao. J mol struct. 2014;1076:535-8.

39. Soares GL, Santos CS, Freitas JCR, Oliveira WA. Síntese e avaliação do prop-2-in-1-il 4,6-di-Oacetil-2,3-didesoxi- $\alpha$-D-eritro-hex-2-

enopiranosídeo contra espécies não albicans de Candida e resultados da associação com a anfotericina B ou com o cetoconazol Rev PanAmaz Saude. 2018;9:43-50.

\section{CONFLITO DE INTERESSES}

Os autores declaram não haver conflitos de interesse.

\section{AUTOR PARA CORRESPONDÊNCIA}

\section{Rodrigo Ribeiro Alves Caiana}

Centro de Educação e Saúde

Universidade Federal de Campina Grande - UFCG

Olho D'Agua da Bica S/N

58175-000, Cuité - PB, Brasil

E-mail: rodrigoribeiroalves@ hotmail.com

Submetido em 27/11/2019

Aceito em 20/04/2020 